\title{
Transcranial Magnetic Stimulation for Post-Operative Neurorehabilitation in Neuro-Oncology: A Review of the Literature and Future Directions
}

Evan Hy Einstein ( $\square$ eeinstein@northwell.edu )

Lenox Hill Hospital https://orcid.org/0000-0001-6489-4128

Nicholas B. Dadario

UMDNJ Robert Wood Johnson Medical School: Rutgers Robert Wood Johnson Medical School

Hamza Khilji

Lenox Hill Hospital

Justin W. Silverstein

Lenox Hill Hospital

Michael E. Sughrue

Prince of Wales Hospital

Randy S. D'Amico

Lenox Hill Hospital

Research Article

Keywords:

Posted Date: February 7th, 2022

DOI: https://doi.org/10.21203/rs.3.rs-1328341/v1

License: (c) (1) This work is licensed under a Creative Commons Attribution 4.0 International License. Read Full License

Version of Record: A version of this preprint was published at Journal of Neuro-Oncology on March 25th, 2022. See the published version at https://doi.org/10.1007/s11060-022-03987-9. 


\section{Abstract}

Introduction: Transcranial magnetic stimulation (TMS) is a neuromodulation technology capable of targeted stimulation and inhibition of cortical areas. Repetitive TMS (rTMS) has demonstrated efficacy in the treatment of several neuropsychiatric disorders, and novel uses of rTMS for neurorehabilitation in patients with acute and chronic neurologic deficits are being investigated. However, studies to date have primarily focused on neurorehabilitation in stroke patients, with little data supporting its use for neurorehabilitation in brain tumor patients.

Methods: We performed a review of the current available literature regarding uses of rTMS for neurorehabilitation in post-operative neuro-oncologic patients.

Results: Data have demonstrated that rTMS is safe in the post-operative neuro-oncologic patient population, with minimal adverse effects and no documented seizures. The current evidence also demonstrates potential effectiveness in terms of neurorehabilitation of motor and language deficits.

Conclusions: Although data are overall limited, both safety and effectiveness have been demonstrated for the use of rTMS for neurorehabilitation in the neurooncologic population. More randomized controlled trials and specific comparisons of contralateral versus ipsilateral rTMS protocols should be explored. Further work may also focus on individualized, patient-specific TMS treatment protocols for optimal functional recovery.

\section{Introduction}

Transcranial magnetic stimulation (TMS) is a non-invasive brain stimulation tool that utilizes magnetic currents to induce electrical activity capable of depolarizing targeted cortical regions [1, 2]. Importantly, TMS can be applied to precisely targeted brain regions using commercially available frameless stereotactic techniques. Furthermore, the generated electrical field can be modulated based on magnetic pulse waveform, frequency of stimulation, pattern of stimulation, as well as variables such as the orientation of the current lines induced in the brain and excitable neural elements [3]. These elements permit tailored, patient-specific stimulation paradigms.

Three types of general TMS protocols exist, including single-pulse, paired pulse, and repetitive TMS (rTMS). In particular, rTMS is used to facilitate excitation or inhibition of cortical areas and is often used in research related to treatment modalities. In rTMS, multiple single-pulse stimuli are delivered at a specified time duration, frequency, and intensity with effects varying according to stimulation parameters [4]. Slow rTMS, for example at $1 \mathrm{~Hz}$ or one magnetic pulse per second, has demonstrated inhibitory effects. In comparison, fast rTMS at 10 or $20 \mathrm{~Hz}$, has demonstrated excitatory effects [5-7].

Substantial research has explored potential applications of TMS for a multitude of neurological and neuropsychiatric conditions. Depression has been the most studied condition overall [8-11]. Specifically, high-frequency rTMS of the left dorsolateral prefrontal cortex (DLPFC) was shown in multiple studies to improve depressive symptoms, and was ultimately FDA approved in 2008 for patients with depression resistant to one antidepressant medication trial [4]. Evidence also exists for the use of rTMS in conditions such as pain, movement disorders, tinnitus, obsessive-compulsive disorder (OCD), schizophrenia, addiction, and other disorders of consciousness [2].

The concept of using rTMS for neurorehabilitation is not novel and has primarily been evaluated in patients after stroke. TMS protocols for post-stroke neurorehabilitation typically involve a patterned protocol known as theta burst stimulation (TBS), which has demonstrated relatively few adverse effects as compared to more conventional rTMS protocols [12]. These protocols are further divided into either delivering low-frequency, inhibitory TBS to the contralateral hemisphere (continuous TBS; cTBS) or high-frequency, stimulatory TBS to the ipsilateral affected hemisphere (intermittent TBS; iTBS). Data from these studies have suggested that downregulation of excitability of the intact or contralateral hemisphere by using TBS on a case-by-case basis results in improvements in paresis, language, attention, memory, and somatosensory processing when combined with physical rehabilitation [13, 14]. In addition, ipsilateral excitatory TBS has also been demonstrated to improve motor recovery $[15,16]$. These data support the efficacy of rTMS improve motor [17] and language [18] recovery following stroke.

Given the evidence for using rTMS for neurorehabilitation after stroke, there is reason to believe that this technology could be beneficial post-operatively for recovery in patients with brain tumors. TMS has been used pre-operatively for cortical mapping of language and motor functions in neurosurgical patients with demonstrated tolerability and safety [19]. However, there is currently a paucity of data to illustrate post-operative efficacy related to neurorehabilitation and recovery. We review these currently limited data and discuss future directions for the use of rTMS for neurorehabilitation in neuro-oncologic patients with pre- and post-operative deficits as a result of their tumor or surgical treatment.

\section{Methods}

A PubMed search was performed using the phrase "transcranial magnetic stimulation brain tumor post-operative" and included articles up to December 2021. This yielded 16 results with all abstracts reviewed. Only two articles described using rTMS to improve function and rehabilitation post-operatively. A further search was performed using another phrase "transcranial magnetic stimulation postsurgical", which produced 19 more abstracts which were reviewed. One double-blinded randomized controlled trial was found for post-operative rTMS use in neuro-oncology patients, which was therefore included in this review. A final search was conducted using the phrase "rTMS stimulation brain tumor", which delivered 288 results. Titles were reviewed and one article was found for inclusion in this review. Lastly, we were alerted to unpublished data from another institution demonstrating a proof-of-concept rTMS paradigm for postoperative neuro-oncologic patients. This was also included in our review with their permission.

\section{Post-Operative TMS in Neuro-Oncologic Patients}

Search Results are presented in Table 1. We identified three case reports involving four total patients [20-22], one recent randomized double-blinded shamcontrolled trial involving 21 patients [23], and one proof-of-concept study involving 31 patients [24].

Page 2/9 
In the first case report [20], a patient with a left subfrontal glioma underwent surgery with residual right upper extremity hemiparesis that was stable at 5 years after surgery. Contralateral inhibitory TBS was used to target the right primary motor cortex without navigation. Low frequency $1 \mathrm{~Hz}$ rTMS was used with each session consisting of 1200 pulses. The optimal stimulation site was determined by the largest motor evoked potentials of the first dorsal interosseous (FDI) muscle of the left upper limb. Intensity of stimulation was set at $90 \%$ of motor threshold of the FDI. Patient did experience headache and nausea during week 3 of treatment, but otherwise no other adverse effects or worsening neurological symptoms were noted with treatment. The patient underwent 22 daily sessions and experienced immediate improvement in right upper extremity function with treatment as well as further improvement upon follow up four weeks after final treatment.

In a second case report [21], a patient with a left precentral oligodendroglioma underwent awake craniotomy and subtotal resection given tumoral involvement in Broca's area. Tumor progression was noted nine months post-operatively, with worsening expressive aphasia. At that time, ipsilateral excitatory TBS was administered daily for 12 days to Broca's area. A BrainSight neuronavigator (Rogue Research, Inc., Montreal, Canada) was used to direct the center of stimulation at the posterior part of the inferior frontal gyrus just anterior to the tumor, where functional MR imaging had demonstrated Broca's area activation. Stimulation parameters involved a power of $60 \%$, frequency of $45 \mathrm{~Hz}$, three pulses, five bursts, 40 cycles, with a cycle duration of 1.0 sections. The patient's repetition and nomination worsened immediately after each rTMS session but improved over basal values after intensive language rehabilitation following each session. The study found that overall basal values improved globally along the experiment but with diminishing returns after each procedure. Interpretation of the results were difficult given tumor progression concurrent with rehabilitation efforts.

In a third case report [22], two patients underwent contralateral inhibitory rTMS to the right M1 area in one patient, and the right inferior frontal gyrus in the other immediately post-operatively after resection of a left insular anaplastic astrocytoma and left temporal glioblastoma, respectively (Table 1). Navigated TMS using a MagVenture MagPro device (MagVenture, Alpharetta, GA) was initiated for both patients during the immediate post-operative period within 24-48 hours during their hospital stay. Both patients received slow rTMS of $5 \mathrm{~Hz}$ with 200 total pulses at $80 \%$ of motor threshold. One patient received one total treatment and the other patient received three treatments. Neither patient demonstrated improvements with treatment in motor function or aphasia and were subsequently lost to follow up. While the results of this study were not supportive of the rehabilitative potential of rTMS, the authors noted that no adverse reactions or events occurred, and no neurologic worsening was noted. Most notably, no seizures were elicited, despite one of the patients having a known seizure disorder as a result of her brain tumor.

Using these data and with support from neurorehabilitation data in stroke, a randomized controlled trial included 21 patients that had undergone surgical intervention for glioma complicated by persistent surgery-related upper extremity paresis. A total of 15 patients underwent contralateral inhibitory navigated rTMS to motor regions based on mapping, and six patients underwent sham procedures [23]. All patients were suffering from acute surgery-related paresis of the upper extremity after glioma resection. The study group protocol involved daily rTMS sessions for seven days at a low frequency of $1 \mathrm{~Hz}$ for 15 minutes totaling 900 pulses at an intensity of $110 \%$ of resting motor potential. Compared with the group undergoing sham treatment, the rTMS group demonstrated significantly improved outcomes on Fugl-Meyer Assessment (FMA) and using the National Institutes of Health Stroke Scale (NIHSS). This overall suggests the potential for use of contralateral inhibitory rTMS for improvement of acute post-operative motor deficits.

Most recently, a proof-of-concept study demonstrated the safety profile and feasibility in using brain network guided rTMS to promote functional recovery immediately after glioma surgery in 31 patients with motor and language deficits post-craniotomy [24]. Unlike previous work, this study utilized a data-driven approach for agile target selection based on individualized brain connectivity analyses. Based on previous work demonstrating the use of cTBS to induce cortical depression and iTBS to induce cortical excitability [25], the authors included a mixture of both cTBS and iTBS protocols based on patient-specific connectivity abnormalities which were identified as compared to a normative healthy atlas of functional connectivity data (Fig 1). Thus, abnormally hypoconnected regions in the ipsilateral hemisphere were often treated with iTBS to increase functional compensation, while cTBS facilitated decreased transcallosal inhibition when used on abnormally hyperconnected regions in the contralateral hemisphere. This novel protocol based on individualized connectomic data was applied within 2 weeks after glioma surgery and resulted in statistically significant improvements in both motor $(p<0.001)$ and language $(p=0.001)$ functions. Importantly, there were no instances of seizure reported and adverse events were limited to headache in four patients.

\section{Discussion}

Neurorehabilitation after stroke using rTMS has been studied through several randomized controlled trials and meta-analyses for treatment of both acute and chronic motor and language deficits with positive results [13-18]. Surgical intervention for brain tumors, particularly when involving eloquent regions, carries a risk of neurologic complications with deficits involving motor and speech function. These deficits may be due to surgical damage to critical cortical and subcortical pathways, vascular injury, or disruption of critical network connections involved in complex neurologic functions. Evidence has supported the ability of rTMS to map eloquent regions involved in motor and speech function preoperatively to help guide intraoperative identification and thus preservation of these functional pathways [19]. However, there is limited evidence for rTMS neurorehabilitation for motor and speech deficits acquired post-operatively in patients with brain tumors. Given the efficacy in stroke, investigations into the potential of targeted rTMS to improve post-operative outcomes in brain tumor patients has been pursued with promising results $[26,27]$. We reviewed the current literature for data involving post-operative rTMS use for neurorehabilitation.

A review of the published literature through 2021 yielded five studies and a total number of 50 patients who received rTMS post-operatively (Table 1). Techniques varied with 4/5 studies using frameless stereotactic navigation for targeting. In addition, stimulation protocols varied, but mostly involved contralateral inhibitory stimulation of the primary motor cortex for motor rehabilitation. Most commonly, a frequency of $1-5 \mathrm{~Hz}$ and an intensity of $80-90 \%$ of motor threshold was utilized in conjunction with intense physical therapy and rehabilitation. Studies varied widely on the timeframe of initiating TMS postoperatively, but most studies prescribed a course of rTMS once daily for between 5 to 22 days. Heterogeneity exists regarding when to start treatment relative to surgery. 
Importantly, post-operative rTMS was shown to be safe in the neuro-oncology population, as only five total patients experienced transient headache and no other adverse effects were noted, including wound issues. While the risk of seizures is the most severe known adverse effect of TMS, and although several cases have been reported to date, risk of seizure is believed to be less than $1 \%$ [28]. Recent larger studies with glioma patients have demonstrated zero instances of seizure following rTMS post-operatively [23, 24]. TBS specifically has been associated with a lower estimated risk of 0.02\% [29], and TBS protocols are therefore better suited to neurosurgical patients in general. The lack of seizures or any significant adverse effects after TMS use in brain tumor patients is further reassuring given that these patients are seen to be at higher risk for seizures. In one case report, one patient had known seizures related to her brain tumor and TMS did not induce any seizures while she was maintained on her anti-epileptic medication regimen [22]. However, the overall risk must be evaluated on a case-by-case basis.

In terms of efficacy, rTMS in post-operative neuro-oncology patients did demonstrate benefits for both language and motor recovery. Overall data demonstrate a rate of $90 \%$ reported improvement of some motor or language function. These benefits were primarily shown when using a contralateral inhibitory TBS protocol for treatment of motor deficits, where rTMS was applied to the unaffected hemisphere. However, no comparative data exists in the neuro-oncology literature between iTBS or cTBS making it hard to draw conclusions on optimized treatment. One case report used ipsilateral stimulatory TBS for rehabilitation of expressive aphasia that did demonstrate global improvement but with diminishing recovery benefits after each rTMS session [21]. These data are difficult to interpret, as deficit progression was concurrent with tumor progression within eloquent language regions. Nonetheless, these data are interesting as language function tends to be lateralized and contralateral inhibitory stimulation may not have the same benefits as with motor function. Further study into brain network guided rTMS may provide insight into the choice of using cTBS or iTBS in select cases. Specifically, given the complexity of language production, further research into ipsilateral excitatory stimulation versus network stimulation in patients with language dysfunction is warranted.

In the only published randomized controlled trial, contralateral TBS was used daily for 7 days post-operatively, but immediate changes in motor function were not statistically significant [23]. However, after three months, statistically significant motor recovery was demonstrated as compared to sham controls. Although benefits were indeed seen immediately following TMS treatments, it appears these benefits may only become significant after several weeks or months. These data are difficult to interpret however given the natural potential for recovery following surgery in close proximity, but without disruption of eloquent regions.

\section{Future Directions: Network Guided Approach for Individualized Targeting}

Given the published support for the safety of rTMS-assisted neurorehabilitation following surgical management for brain tumors, future studies should be designed to better understand the potential efficacy of the treatment as well to better define treatment algorithms that optimize this efficacy. These studies are already ongoing in the stroke rehabilitation literature [30] and neuropsychiatric patients [31]. From previous work, it is clear that the efficacy of rTMS treatment is highly related to the individual targets selected [32] as well as the precision in modulating those specific targets [33]. Given that specific symptoms likely localize to specific underlying connections within brain networks, these concerns may be better addressed moving forward with improved consideration of patient-specific brain connectivity [34]. Importantly, while patients may both present with the same post-operative functional deficits, they may require patientspecific targets to modulate specific networks [32] or even specific connectivity abnormalities [35]. Furthermore, while many non-invasive stimulation protocols rely on standard craniometric measurements, millimeter differences over a patient's scalp may selectively modulate completely different cortical subcortical connections that are unwarranted. However, an individualized neuroimaging-based approach which utilizes anatomically fine, parcel-guided rTMS to treat patient-specific connectivity abnormalities (Fig 2) provides one way to treat pathophysiologic signature profiles of patient-specific symptoms postoperatively more effectively $[24,36]$.

\section{Limitations}

As the current published data regarding rTMS-assisted neurorehabilitation for brain tumor patients has been derived from case reports and limited series, interpretation of the potential efficacy of rTMS remains challenging. Additionally, natural post-operative neural reorganization processes with time may act as a confounding factor to these reports of motor and language recovery after TMS. As with stroke, which results in a fixed deficit, tumor progression may further compromise function and thus limit recovery efforts. While data from randomized controlled trials in stroke patients are otherwise promising, there is a need to address this factor in the post-operative neuro-oncologic population. This therefore calls for more randomized controlled trials in this population. Furthermore, the limited data led to challenges in quantifying the benefits of different parameters, though it does appear contralateral inhibitory TBS has been used successfully more frequently for motor deficit recovery. Future work should involve comparisons of different parameters and rTMS protocols to determine the most effective post-operative recovery modality.

\section{Conclusions}

There is recent developing evidence of using rTMS for neurorehabilitation in post-operative neuro-oncologic patients. Although limited data exist, the cases demonstrate safety and potential effectiveness for post-operative motor and language recovery. Further studies including randomized sham-controlled trials will allow for further evaluation of possible benefits.

\section{Declarations}

Funding: The authors declare that no funds, grants, or other support were received during the preparation of this manuscript.

Competing Interests: The authors have no relevant financial or non-financial interests to disclose. 
Author Contributions: All authors contributed to the preparation of this manuscript. Literature search and results collection were performed by Evan Einstein and Hamza Khilji. Primary writing of the body of the manuscript was performed by Evan Einstein and Randy D'Amico. Several sections were written by Nicholas Dadario and Michael Sughrue. The figures were created by Nicholas Dadario and Michael Sughrue. All authors were involved in the editing and revision of this manuscript. All authors read and approved the final manuscript.

Data Availability: The datasets generated during and/or analyzed during the current study are available from the corresponding author on reasonable request.

Ethics Approval: N/A

Consent to Participate: N/A

Consent to Publish: N/A

\section{References}

1. Merton PA, Morton HB. Stimulation of the cerebral cortex in the intact human subject. Nature. 1980;285(5762):227. 10.1038/285227a0

2. Lefaucheur JP, Andre-Obadia N, Antal A, Ayache SS, Baeken C, Benninger DH, et al. Evidence-based guidelines on the therapeutic use of repetitive transcranial magnetic stimulation (rTMS). Clin Neurophysiol. 2014;125(11):2150-206. 10.1016/j.clinph.2014.05.021

3. Klomjai W, Katz R, Lackmy-Vallee A. Basic principles of transcranial magnetic stimulation (TMS) and repetitive TMS (rTMS). Ann Phys Rehabil Med. 2015;58(4):208-13. 10.1016/j.rehab.2015.05.005

4. Galletta EE, Rao PR, Barrett AM. Transcranial magnetic stimulation (TMS): potential progress for language improvement in aphasia. Top Stroke Rehabil. 2011;18(2):87-91. 10.1310/tsr1802-87

5. Maeda F, Keenan JP, Tormos JM, Topka H, Pascual-Leone A. Modulation of corticospinal excitability by repetitive transcranial magnetic stimulation. Clin Neurophysiol. 2000;111(5):800-5. 10.1016/s1388-2457(99)00323-5

6. Pascual-Leone A, Valls-Sole J, Wassermann EM, Hallett M. Responses to rapid-rate transcranial magnetic stimulation of the human motor cortex. Brain. 1994;117 ( Pt 4):847-58. 10.1093/brain/117.4.847

7. Berardelli A, Inghilleri M, Rothwell JC, Romeo S, Curra A, Gilio F, et al. Facilitation of muscle evoked responses after repetitive cortical stimulation in man. Exp Brain Res. 1998;122(1):79-84. 10.1007/s002210050493

8. George MS, Lisanby SH, Avery D, McDonald WM, Durkalski V, Pavlicova M, et al. Daily left prefrontal transcranial magnetic stimulation therapy for major depressive disorder: a sham-controlled randomized trial. Arch Gen Psychiatry. 2010;67(5):507-16. 10.1001/archgenpsychiatry.2010.46

9. George MS, Wassermann EM, Kimbrell TA, Little JT, Williams WE, Danielson AL, et al. Mood improvement following daily left prefrontal repetitive transcranial magnetic stimulation in patients with depression: a placebo-controlled crossover trial. Am J Psychiatry. 1997;154(12):1752-6. 10.1176/ajp.154.12.1752

10. O'Reardon JP, Solvason HB, Janicak PG, Sampson S, Isenberg KE, Nahas Z, et al. Efficacy and safety of transcranial magnetic stimulation in the acute treatment of major depression: a multisite randomized controlled trial. Biol Psychiatry. 2007;62(11):1208-16. 10.1016/j.biopsych.2007.01.018

11. Avery DH, Isenberg KE, Sampson SM, Janicak PG, Lisanby SH, Maixner DF, et al. Transcranial magnetic stimulation in the acute treatment of major depressive disorder: clinical response in an open-label extension trial. J Clin Psychiatry. 2008;69(3):441-51. 10.4088/jcp.v69n0315

12. Chung SW, Hill AT, Rogasch NC, Hoy KE, Fitzgerald PB. Use of theta-burst stimulation in changing excitability of motor cortex: A systematic review and meta-analysis. Neurosci Biobehav Rev. 2016;63:43-64. 10.1016/j.neubiorev.2016.01.008

13. Hoyer EH, Celnik PA. Understanding and enhancing motor recovery after stroke using transcranial magnetic stimulation. Restor Neurol Neurosci. 2011;29(6):395-409. 10.3233/RNN-2011-0611

14. Hummel FC, Cohen LG. Non-invasive brain stimulation: a new strategy to improve neurorehabilitation after stroke? Lancet Neurol. 2006;5(8):708-12. 10.1016/S1474-4422(06)70525-7

15. Chang WH, Kim YH, Bang OY, Kim ST, Park YH, Lee PK. Long-term effects of rTMS on motor recovery in patients after subacute stroke. J Rehabil Med. 2010;42(8):758-64. 10.2340/16501977-0590

16. Khedr EM, Etraby AE, Hemeda M, Nasef AM, Razek AA. Long-term effect of repetitive transcranial magnetic stimulation on motor function recovery after acute ischemic stroke. Acta Neurol Scand. 2010;121(1):30-7. 10.1111/j.1600-0404.2009.01195.x

17. Xie Y-J, Chen Y, Tan H-X, Guo Q-F, Lau B, Gao Q. Repetitive transcranial magnetic stimulation for lower extremity motor function in patients with stroke: a systematic review and network meta-analysis. Neural Regeneration Research. 2021;16(6):1168-76. 10.4103/1673-5374.300341

18. Zhang J, Zhong D, Xiao X, Yuan L, Li Y, Zheng Y, et al. Effects of repetitive transcranial magnetic stimulation (rTMS) on aphasia in stroke patients: A systematic review and meta-analysis. Clin Rehabil. 2021;35(8):1103-16. 10.1177/0269215521999554

19. Tarapore PE, Picht T, Bulubas L, Shin Y, Kulchytska N, Meyer B, et al. Safety and tolerability of navigated TMS for preoperative mapping in neurosurgical patients. Clin Neurophysiol. 2016;127(3):1895-900. 10.1016/j.clinph.2015.11.042

20. Kakuda W, Abo M, Kobayashi K, Momosaki R, Yokoi A, Ito H, et al. Low-frequency rTMS combined with intensive occupational therapy for upper limb hemiparesis after brain tumour resection. Brain Injury. 2010;24(12):1505-10. 10.3109/02699052.2010.523040

21. Barcia JA, Sanz A, González-Hidalgo M, de Las Heras C, Alonso-Lera P, Díaz P, et al. rTMS stimulation to induce plastic changes at the language motor area in a patient with a left recidivant brain tumor affecting Broca's area. Neurocase. 2012;18(2):132-8. 10.1080/13554794.2011.568500 
22. O'Neal CM, Stephens TM, Briggs RG, Sughrue ME, Conner AK. Navigated transcranial magnetic stimulation following awake craniotomy for resection of glioma: Description of two cases. Surg Neurol Int. 2020;11:433. 10.25259/SNI_628_2020

23. Ille S, Kelm A, Schroeder A, Albers LE, Negwer C, Butenschoen VM, et al. Navigated repetitive transcranial magnetic stimulation improves the outcome of postsurgical paresis in glioma patients - A randomized, double-blinded trial. Brain Stimul. 2021;14(4):780-7. 10.1016/j.brs.2021.04.026

24. Poologaindran A, Profyris C, Young IM, Dadario NB, Ahsan SA, Chendeb K, et al. Interventional Neurorehabilitation for Promoting Functional Recovery Post-Craniotomy: A Proof-of-Concept. medRxiv. 2021:2021.07.27.21260088. 10.1101/2021.07.27.21260088

25. Huang YZ, Edwards MJ, Rounis E, Bhatia KP, Rothwell JC. Theta burst stimulation of the human motor cortex. Neuron. 2005;45(2):201-6. 10.1016/j.neuron.2004.12.033

26. Lavrador JP, Ghimire P, Brogna C, Furlanetti L, Patel S, Gullan R, et al. Pre- and Intraoperative Mapping for Tumors in the Primary Motor Cortex: DecisionMaking Process in Surgical Resection. J Neurol Surg A Cent Eur Neurosurg. 2021;82(4):333-43. 10.1055/s-0040-1709729

27. Hendrix P, Senger S, Simgen A, Griessenauer CJ, Oertel J. Preoperative rTMS Language Mapping in Speech-Eloquent Brain Lesions Resected Under General Anesthesia: A Pair-Matched Cohort Study. World Neurosurg. 2017;100:425-33. 10.1016/j.wneu.2017.01.041

28. Rossi S, Antal A, Bestmann S, Bikson M, Brewer C, Brockmoller J, et al. Safety and recommendations for TMS use in healthy subjects and patient populations, with updates on training, ethical and regulatory issues: Expert Guidelines. Clin Neurophysiol. 2021;132(1):269-306.

10.1016/j.clinph.2020.10.003

29. Stultz DJ, Osburn S, Burns T, Pawlowska-Wajswol S, Walton R. Transcranial Magnetic Stimulation (TMS) Safety with Respect to Seizures: A Literature Review. Neuropsychiatr Dis Treat. 2020;16:2989-3000. 10.2147/NDT.S276635

30. Hsu W-Y, Cheng C-H, Liao K-K, Lee I-H, Lin Y-Y. Effects of Repetitive Transcranial Magnetic Stimulation on Motor Functions in Patients With Stroke. Stroke. 2012;43(7):1849-57. doi:10.1161/STROKEAHA.111.649756

31. Hausmann A, Kemmler G, Walpoth M, Mechtcheriakov S, Kramer-Reinstadler K, Lechner T, et al. No benefit derived from repetitive transcranial magnetic stimulation in depression: a prospective, single centre, randomised, double blind, sham controlled "add on" trial. J Neurol Neurosurg Psychiatry. 2004;75(2):320-2.

32. Siddiqi SH, Taylor SF, Cooke D, Pascual-Leone A, George MS, Fox MD. Distinct Symptom-Specific Treatment Targets for Circuit-Based Neuromodulation. Am J Psychiatry. 2020;177(5):435-46. 10.1176/appi.ajp.2019.19090915

33. Rosen AC, Bhat JV, Cardenas VA, Ehrlich TJ, Horwege AM, Mathalon DH, et al. Targeting location relates to treatment response in active but not sham rTMS stimulation. Brain Stimul. 2021;14(3):703-9. 10.1016/j.brs.2021.04.010

34. Dadario NB, Brahimaj B, Yeung J, Sughrue ME. Reducing the Cognitive Footprint of Brain Tumor Surgery. Front Neurol. 2021;12:711646. 10.3389/fneur.2021.711646

35. Ren H, Zhu J, Su X, Chen S, Zeng S, Lan X, et al. Application of Structural and Functional Connectome Mismatch for Classification and Individualized Therapy in Alzheimer Disease. Front Public Health. 2020;8:584430. 10.3389/fpubh.2020.584430

36. Moreno-Ortega M, Kangarlu A, Lee S, Perera T, Kangarlu J, Palomo T, et al. Parcel-guided rTMS for depression. Translational Psychiatry. 2020;10(1):283. $10.1038 / \mathrm{s} 41398-020-00970-8$

\section{Tables}

Table 1. Currently published cases of rTMS use in post-operative patients with brain tumors. 


\begin{tabular}{|c|c|c|c|c|c|c|c|c|c|c|}
\hline Study & $\begin{array}{l}\text { Study } \\
\text { Type }\end{array}$ & $\begin{array}{l}\text { \# of } \\
\text { Patients }\end{array}$ & Tumor & Deficit & $\begin{array}{l}\text { Type of } \\
\text { rTMS }\end{array}$ & Protocol & Target & $\begin{array}{l}\text { Additional } \\
\text { Therapies }\end{array}$ & Outcome & $\begin{array}{l}\text { Adver } \\
\text { Effect }\end{array}$ \\
\hline [20] & $\begin{array}{l}\text { Case } \\
\text { Report }\end{array}$ & 1 & $\begin{array}{l}\text { Left subfrontal } \\
\text { glioma }\end{array}$ & $\begin{array}{l}\text { Right } \\
\text { upper } \\
\text { extremity }\end{array}$ & cTBS & $\begin{array}{l}1 \\
\text { session/day } \\
\text { for } 22 \text { days; } \\
1 \mathrm{~Hz} ; 1200 \\
\text { pulses each } \\
\text { session }\end{array}$ & $\begin{array}{l}\text { Right } \\
\text { primary } \\
\text { motor cortex }\end{array}$ & $\begin{array}{l}\text { Intensive } \\
\text { occupational } \\
\text { therapy }\end{array}$ & $\begin{array}{l}\text { Improved } \\
\text { motor } \\
\text { function after } \\
\text { treatment } \\
\text { with further } \\
\text { improvement } \\
4 \text { weeks post- } \\
\text { treatment }\end{array}$ & $\begin{array}{l}\text { Headc } \\
\text { and } n \\
\text { durinc } \\
\text { week } \\
\text { treatr }\end{array}$ \\
\hline [21] & $\begin{array}{l}\text { Case } \\
\text { Report }\end{array}$ & 1 & $\begin{array}{l}\text { Left precentral } \\
\text { oligodendroglioma }\end{array}$ & $\begin{array}{l}\text { Expressive } \\
\text { aphasia }\end{array}$ & iTBS & $\begin{array}{l}1 \\
\text { session/day } \\
\text { for } 12 \text { days; } \\
5 \mathrm{~Hz} ; 40 \\
\text { cycles, cycle } \\
\text { duration } 1.0 \\
\mathrm{~s}\end{array}$ & Broca's area & $\begin{array}{l}\text { Intensive } \\
\text { language } \\
\text { rehabilitation }\end{array}$ & $\begin{array}{l}\text { Global } \\
\text { improvement } \\
\text { but } \\
\text { increasingly } \\
\text { less each } \\
\text { procedure }\end{array}$ & None \\
\hline \multirow[t]{2}{*}{ [22] } & $\begin{array}{l}\text { Case } \\
\text { Report }\end{array}$ & 2 & $\begin{array}{l}\text { Left insular } \\
\text { anaplastic } \\
\text { astrocytoma }\end{array}$ & $\begin{array}{l}\text { Right- } \\
\text { sided } \\
\text { weakness } \\
\text { and } \\
\text { pronator } \\
\text { drift }\end{array}$ & cTBS & $\begin{array}{l}1 \text { total } \\
\text { treatment; } 5 \\
\text { Hz; } 200 \\
\text { pulses total }\end{array}$ & Right M1 & None & $\begin{array}{l}\text { No immediate } \\
\text { changes; mild } \\
\text { improvement } \\
\text { in strength at } \\
4 \text { weeks, then } \\
\text { lost to follow } \\
\text { up }\end{array}$ & None \\
\hline & & & $\begin{array}{l}\text { Left temporal } \\
\text { glioblastoma } \\
\text { multiforme }\end{array}$ & $\begin{array}{l}\text { Expressive } \\
\text { aphasia }\end{array}$ & cTBS & $\begin{array}{l}3 \text { total } \\
\text { treatments; } 5 \\
\mathrm{~Hz} \mathrm{/} 50 \mathrm{~Hz} ; \\
200 \text { pulses } \\
\text { total }\end{array}$ & $\begin{array}{l}\text { Right inferior } \\
\text { frontal gyrus }\end{array}$ & None & $\begin{array}{l}\text { No immediate } \\
\text { change; then } \\
\text { lost to follow } \\
\text { up }\end{array}$ & None \\
\hline [23] & $\mathrm{RCT}$ & $\begin{array}{l}15 \text { in } \\
\text { rTMS } \\
\text { group, } 6 \text { in } \\
\text { sham } \\
\text { group }\end{array}$ & $\begin{array}{l}\text { Glioma, varying } \\
\text { hemispheres and } \\
\text { locations }\end{array}$ & $\begin{array}{l}\text { Surgery- } \\
\text { related } \\
\text { paresis of } \\
\text { upper } \\
\text { extremity }\end{array}$ & cTBS & $\begin{array}{l}1 \\
\text { session/day } \\
\text { for } 7 \text { days } \\
\text { post- } \\
\text { operatively; } 1 \\
\text { Hz for } 15 \\
\text { minutes (900 } \\
\text { pulses) }\end{array}$ & $\begin{array}{l}\text { Contralateral } \\
\text { motor } \\
\text { hotspot } \\
\text { based on } \\
\text { motor } \\
\text { mapping }\end{array}$ & PT & $\begin{array}{l}\text { Immediate } \\
\text { changes were } \\
\text { not } \\
\text { statistically } \\
\text { significant but } \\
\text { improvements } \\
\text { at 3-month } \\
\text { follow up } \\
\text { were } \\
\text { statistically } \\
\text { significant vs } \\
\text { sham group }\end{array}$ & None \\
\hline [24] & $\begin{array}{l}\text { Proof- } \\
\text { of- } \\
\text { concept }\end{array}$ & $\begin{array}{l}31 \\
\text { patients } \\
\text { underwent } \\
\text { rTMS } \\
\text { post- } \\
\text { operatively }\end{array}$ & $\begin{array}{l}\text { Glioma, varying } \\
\text { hemispheres and } \\
\text { locations }\end{array}$ & $\begin{array}{l}\text { Variety of } \\
\text { post- } \\
\text { operative } \\
\text { motor and } \\
\text { language } \\
\text { deficits }\end{array}$ & Variable & $\begin{array}{l}5 \\
\text { sessions/day } \\
\text { for } 5 \text { days; } \\
\text { varying } \\
\text { protocols per } \\
\text { patient } \\
\text { deficit }\end{array}$ & Varying & $\begin{array}{l}\text { PT or speech } \\
\text { rehabilitation }\end{array}$ & $\begin{array}{l}\text { Observed } \\
\text { benefit in } \\
28 / 31 \\
\text { patients }\end{array}$ & $\begin{array}{l}4 / 31 \\
\text { experi } \\
\text { transi } \\
\text { heada } \\
\text { no ott } \\
\text { adver: } \\
\text { events }\end{array}$ \\
\hline
\end{tabular}

\section{Figures}



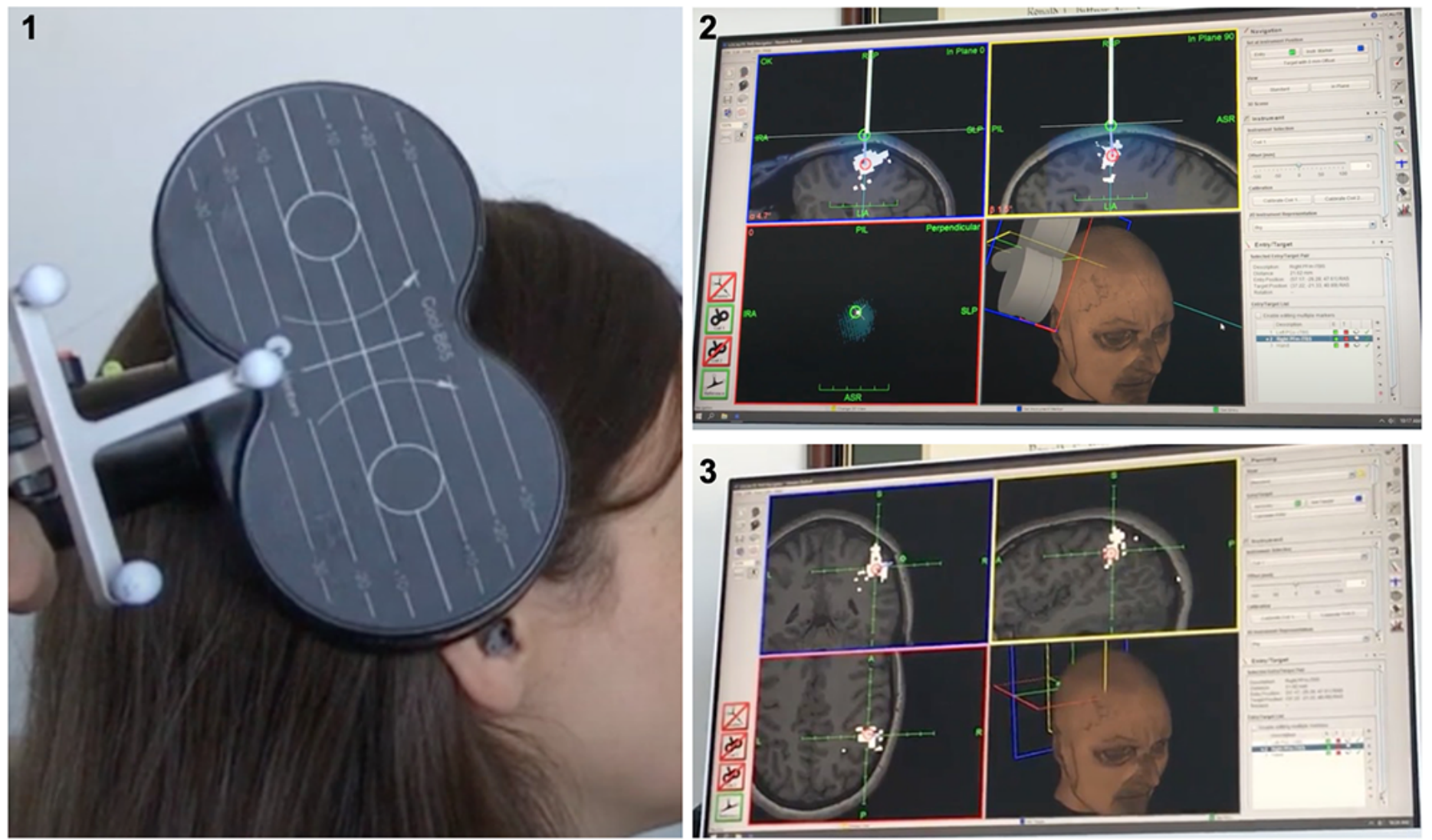

Figure 1

Demonstration of Image-Guided TMS treatment. A butterfly TMS coil is shown in image 1. The coil is placed with computer image guidance to ensure accurate placement over the target (image 2). An example of a target area defined by a precise anatomic cortical parcellation is shown in the image 3 , in which the red area is the target, green is the entry zone, and white is the parcellation

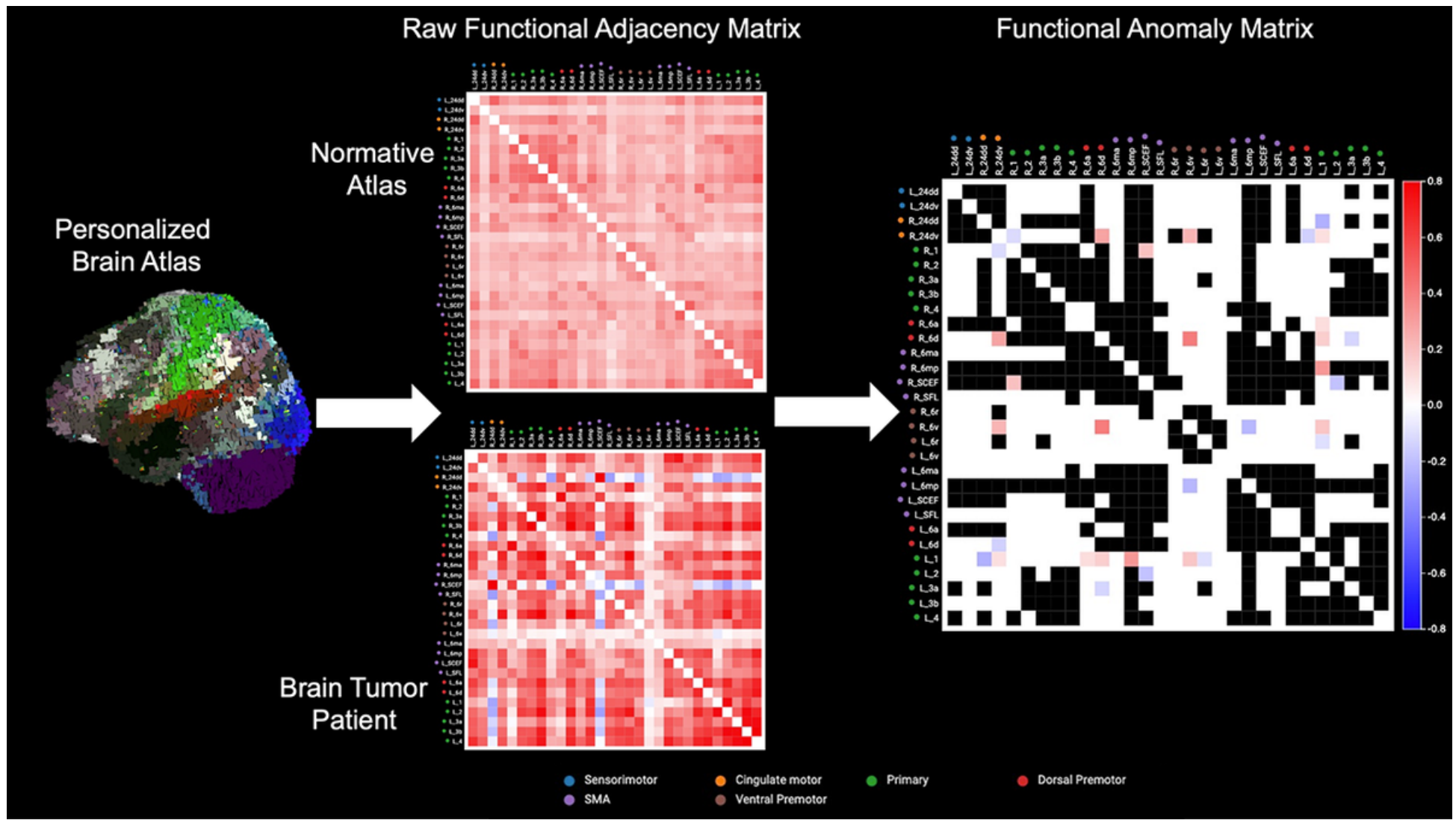


Figure 2

Individualized TMS Treatment According to Connectomic Guided Targeting. The current figure demonstrates the feasibility of utilizing personalized connectomic data to define patient-specific TMS targets according to network abnormalities in a brain tumor patient after surgery. Individualized brain connectivity mapping is performed on the patient utilizing an anatomically specific, surface-based parcellation scheme (first column). Functional connectivity adjacency matrices are created for the patient and compared to healthy patients in a normative atlas (second column). These raw functional connectivity matrices are compared to identify individual outlier parcels for the patient, or functional connectivity "anomalies" which are parcels defined as functioning significantly outside of the normal range. Individual anomalies identified represent positive (red) and negative (blue) correlations between two BOLD signals between two individual parcels (third column). These anomalies represent possible rehabilitation targets to re-synchronize a dysfunctional network which may have been damaged during surgery. Here, various motor networks are shown (represented by different colors) for possible targets in a patient with hemiparesis following surgical resection. Individual parcellations in the current matrices are defined according to the previously published Human Connectome Project atlas.

Abbreviations: SMA, supplementary motor area 